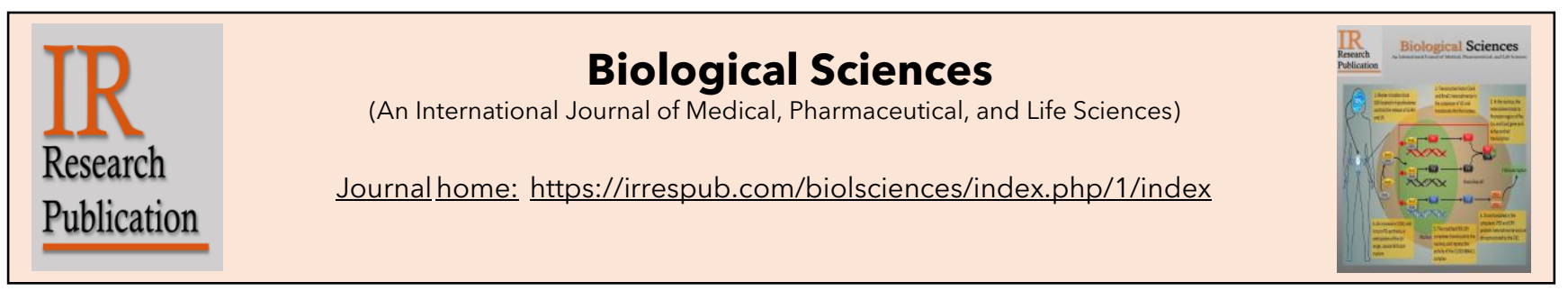

\title{
Optimization of growth parameters for enhancing antifungal secondary metabolites of Talaromyces islandicus VSGF1 against drug resistant Candida spp.
}

\author{
Sangeeta M. Kamradgi, Vidyasagar M. Gunagambhire* \\ Microbiology and Medicinal Plant Laboratory, Department of PG Studies and Research in Botany, Gulbarga University, \\ Kalaburagi-585106, Karnataka, India.
}

\section{ARTICLE HISTORY}

Received: 30-04-2021

Revised: 05-05-2021

Accepted: 05-05-2021

Online: 08-05-2021

\section{KEYWORDS}

Antifungal activity

Candida spp.

Growth parameters

Secondary metabolites

Talaromyces islandicus

\section{ABSTRACT}

The rapid increase in resistance to antifungal drugs has been raised largely in Candida species due to continue and over utilization of available frontline drugs. To overcome this rising health care issue, it's necessary to identify a new and improved antifungal drug without causing any side effects than the existing drugs. In view of this, the present investigation was carried out in findings of the novel antifungal drug against pathogenic Candida species. The fungus Talaromyces islandicus VSGF1 was screened for the production of antifungal secondary metabolites through optimization of growth parameters under submerged fermentation by changing one variable at a time. The highest fungal biomass $(1.9 \mathrm{~g} / 100 \mathrm{ml}$ of culture broth) with significant antifungal activity (15 $\mathrm{mm}$ zone of inhibition) was achieved in potato dextrose broth supplemented with $2 \%$ dextrose at $\mathrm{pH} 6$ and $26 \mathrm{OC}$ temperature at stationary condition. A comparative antifungal activity was performed on frontline drugs of amphotericin B, fluconazole, ketoconazole, and nystatin along with extracellular and intracellular crude extracts of $T$. islandicus against eight Candida strains. Among the frontline drugs, only ketoconazole exhibited antifungal activity against tested Candida spp. and resistance has been observed by amphotericin B, fluconazole, and nystatin. Interestingly, both extracts of $T$. islandicus were found to possess significant and a broad spectrum of inhibitory activity against all tested Candida spp. This is the first report on this species from the genus Talaromyces that was used for the optimization of process parameters and contribution to the potential producers for antifungal secondary metabolites.
*Address for correspondence

Microbiology and Medicinal Plant Laboratory, Department of PG Studies and Research in Botany, Gulbarga University, Kalaburagi585106, Karnataka, India

Email: gmvidyasagar@gmail.com

DOI: http://dx.doi.org/10.55006/biolsciences.2021.1110

Published by IR Research Publication; Kamradgi SM et al (C) 2021 by Biological Sciences is licensed under CC BY 4.0 cc)

\section{Introduction}

Microbes are evidence for the discovery of pharmaceutical and industrial active natural products. Among the microbial community, fungi are major sources for therapeutic applications for human welfare have been proven from many years. The genus Aspergillus and Penicillium are the most 
Abbreviations: g/L: Gram's per liter; mm: Milliliter; MTCC: Microbial type culture collection; OC: Temperature; RPM: Rotation per minute; SMF: Submerged fermentation; spp: Species; SSF: Solid state fermentation; ZOI: Zone of inhibition.

dominant fungi frequently reported for the production of novel pharmacological lead metabolites. Besides that, the maximum number of pharmaceutical active metabolites was also reported from Fusarium, Trichoderma and Talaromyces [1]. The recent investigations have been focused on the genus Talaromyces is due to production of 316 novel pharmaceutical bioactive products and characteristic pigments [2,3]. A new diphenylketones and xanthone derivatives are isolated from Talaromyces islandicus EN-501 exhibited antioxidant and antimicrobial activity, respectively against pathogenic bacteria [4]. Such active biomolecules were reported by more than a single species in the genus Talaromyces, but many species have not been screened scientifically and such metabolites are expressed only under unique circumstances. The majority of active metabolites isolated from Talaromyces are most effective against bacterial and fungal pathogens [5-10]. Hence, this raised our interest to carry out research on the production of potential antifungal secondary metabolites from the genus Talaromyces against Candida species.

At present, the invasive Candida species are major pathogen causes for superficial to most disseminated bloodstream infections associated with wide clinical manifestations notably in immunecompromised patients. The available antifungal agents of polyenes (amphotericin B, nystatin), azoles (ketoconazole, fluconazole, itraconazole, isavuconazole, posaconazole, and voriconazole) and the echinocandins (anidulafungin, caspofungin, and micafungin) are generally used to treat the fungal infections. However, the majority of these drugs are ineffective in treating immunecompromised patients due to multi drug resistance (MDR) in most of the Candida species, particularly in non albican Candida species (NAC) such as Candida glabrata, Candida tropicalis, Candida auris and Candida krusei $[11,12]$. A study during 2007-2017 reveals the average isolation rates of $C$. albicans and NAC spp. with $45.8 \%$ and $67.3 \%$, respectively. Among NAC spp., fluconazole resistance was higher in C. krusei $97.3 \%$ followed by C. glabrata $49.5 \%$, C. tropicalis $34.3 \%$ and C. rugosa 33.3\% [13]. Therefore, there is an urgent need to undertake the studies on identification of a new antifungal drug, as numerous microbes have proved to produce molecules with a broad spectrum of antimicrobial activity. Talaromyces is a mold fungus commonly found in stored cereals. It has a highly versatile metabolism characterized by the secretion of numerous biopolymers degrading enzymes, mycotoxins and anthraquinones that altogether offers a wide spectrum of potential industrial applications [14].

In view of this, the present research was carried out on novel antifungal producing secondary metabolites from Talaromyces islandicus VSGF1 against pathogenic Candida species.

\section{Materials and Methods}

\section{Talaromyces islandicus VSGF1}

The fungus T. islandicus VSGF1 (VSGF1; Prescribed laboratory code for fungal isolate) was previously isolated from vermicompost soil of Kalaburagi region, Karnataka-India and screened for the production of antifungal secondary metabolites. The molecular identification confirmed its identity as Talaromyces islandicus through the internal transcribed spacer of the conserved ribosomal DNA. The sequence was submitted to Gen bank accession number MN818685.1 (Figure 1a, b, \& c).

\section{Test organisms}

Eight Candida strains from four species namely Candida albicans MTCC:1966, C. albicans MTCC:2795, C. albicans MTCC:3019, C. glabrata MTCC:3814, C. glabrata MTCC:3981, C. tropicalis MTCC:1406, C. tropicalis MTCC:230 and C. haemulonii MTCC:8303 were used as the test organisms. The clinical isolates were obtained from Microbial Type Culture Collection (MTCC) and Gene Bank, Institute of Medical Technology (IMTECH), Chandigarh, India.

\section{Chemicals}

Agar, Czapek dox agar, Dextrose, Dipotassium phosphate, Ethyl acetate, Ferrous sulphate, Fructose, Glucose, Magnesium, Magnesium sulfate, Maltose, Martin Rose Bengal, Oat meal, Peptone, Potassium chloride, Potassium phosphate, Sodium nitrate, Starch agar, Sucroce, Yeast extract were purchased from Himedia Laboratory Pvt. Ltd. Amphotericin B, Chloramphenicol, Fluconazole, Ketoconazole, and Nystatin were supplied by Sigma Aldrich.

\section{Optimization of Culture Media}




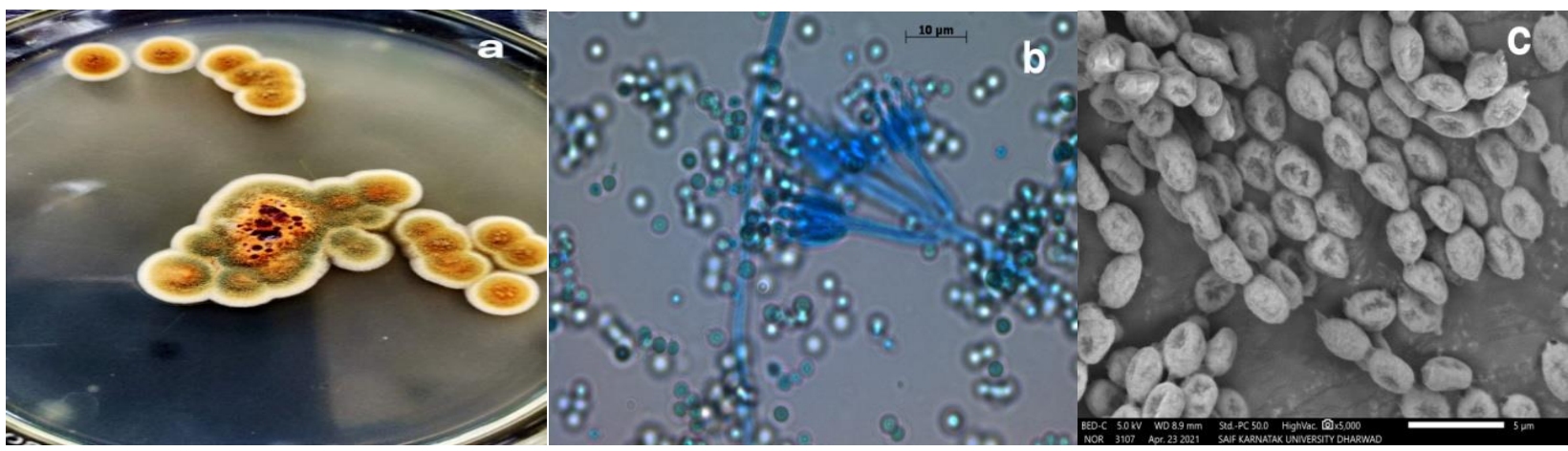

Figure 1. a) Culture plate of $T$. islandicus VSGF1 on potato dextrose agar medium showing colony color, growth pattern and sporulation; inserted image showing reverse plate with pigmentation b) Fungal mycelium with phialides containing conidiophores producing conidia c) chains of single celled conidia

Initially, the culture medium was optimized by using eight different media (Gram's/Liter of distilled water) namely potato dextrose agar (PDA; potato-200g, dextrose-20g, agar-15g), czapek dox agar (CZA; sucroce-30g, sodium nitrate-2g, dipotassium phosphate-1 $\mathrm{g}$, magnesium $0.5 \mathrm{~g}$, potassium chloride- $0.5 \mathrm{~g}$, ferrous sulphate- $0.01 \mathrm{~g}$, agar- $15 \mathrm{~g}$ ), malt extract agar (MEA; malt extract- $30 \mathrm{~g}$, peptone5, agar-15), potato carrot agar (PCA; potato- $200 \mathrm{~g}$, carrot- $250 \mathrm{~g}$, agar-15g), sabouraud dextrose agar (SDA; dextrose-40g, peptone-10, agar-15g), starch agar (SA; 30g), oatmeal agar (OA; oat meal-30g, agar-15g) and Martin Rose Bengal agar media (MRBA; peptone-5, dextrose-10, potassium phosphate-1 $\mathrm{g}$, magnesium sulfate- $0.50 \mathrm{~g}$, rose Bengal-0.05, chloramphenicol-0.10g, agar-15) were used to determine the good growth and sporulation of $T$. islandicus VSGF1 [15]. The freshly sub cultured fungus was inoculated in each petriplate containing different medium was incubated at $28 O C$ and regular observations were made in 7-10 days. The medium which supports the better growth and sporulation were further subjected to broth cultures for extraction of secondary metabolites to screen the antifungal activity against each one of the Candida species.

\section{Optimization of Growth Parameters}

The optimization of culture medium was performed using conventional methods by changing various parameters such as carbon, nitrogen, $\mathrm{pH}$, temperature, incubation period and agitation for the production of highest biomass and antifungal secondary metabolites.

\section{Effect of Carbon Sources}

Various carbon sources such as dextrose, fructose, glucose, maltose (simple carbon) and sucrose (complex carbon) were used for the optimization. The Erlenmeyer flask containing $100 \mathrm{ml}$ of basal medium added with each carbon source (2\% weight/volume) were inoculated with $5 \mathrm{~mm}$ diameter of fungal disc and incubated at $28 \mathrm{OC}$ for 7 days [16]. The biomass was harvested through centrifugation, washed two to three times with deionized water and removed the excess water through blotting filter paper. Then, the biomass was weighed as $\mathrm{g} / 100 \mathrm{ml}$ of broth culture. The filtered broth was treated with an equal volume of ethyl acetate (organic solvent) and the solvent phase was collected and condensed to form a crude extract. The obtained crude extract was subjected to antifungal activity against each one of the Candida spp.

\section{Effect of Nitrogen Sources}

The each $0.5 \%(\mathrm{w} / \mathrm{v})$ of peptone and yeast extract was served as nitrogen sources were added to the basal medium with $2 \%$ of dextrose as carbon source and medium without nitrogen source used as a control [15]. The flasks were inoculated with $5 \mathrm{~mm}$ fungal mycelium and incubated at 28 OC. After 7 days, the amount of biomass and antifungal activity was recorded as mentioned above.

\section{Effect of $\mathrm{pH}$}

The five conical flasks containing $100 \mathrm{ml}$ of basal medium was adjusted to various $\mathrm{pH}$ from $4-8$ by adding $0.1 \mathrm{~N} \mathrm{NaOH}$ or $0.1 \mathrm{~N} \mathrm{HCl}$. The $5 \mathrm{~mm}$ diameter of the fungal mycelium disc was inoculated and incubated at 26 OC [17]. After 7days, the effect of $\mathrm{pH}$ was determined through the weight of biomass production and highest antimicrobial activity.

\section{Effect of Temperature}

The fungus was subjected to different temperature ranges from 25 to $30 \mathrm{OC}$ to examine the optimum temperature required for the growth and yield of antifungal metabolites. $100 \mathrm{ml}$ of basal medium amended with $2 \%$ dextrose was inoculated with $5 \mathrm{~mm}$ diameter of fungal mycelium and incubated at provided temperature [18]. After 7 days of 
incubation, the weight of fungal mycelium and zone of inhibition was recorded.

\section{Effect of Incubation Period}

To study the optimum incubation time, the fungus was inoculated in basal medium and incubated at 26 OC with a different time period of $5,7,9,11,13$ \& 15 days. The fungal growth and production of secondary metabolites were kept under continuous observation [19]. After the incubation period, the amount of biomass and zone of inhibition was assessed.

\section{Effect of Agitation}

Five flasks containing $100 \mathrm{ml}$ of basal medium supplemented with $2 \%$ dextrose was inoculated with $5 \mathrm{~mm}$ disc of fungal mycelium and incubated at 26 OC for 7 days at the agitation speed of 100,120 , $140,160,180$ and 200 rotation per minute (rpm) on a rotary shaker [19]. The flask without agitation kept as control. The weight of biomass and the effect of agitation on antimicrobial activity were determined.

Preparation of Fungal Crude Extracts and Standard Drugs

Talaromyces islandicus was subculture on freshly prepared PDA medium. From this, $5 \mathrm{~mm}$ diameter of mature fungal colony was inoculated in $1000 \mathrm{ml}$ Erlenmeyer flask containing $500 \mathrm{ml}$ of optimized PDB with $\mathrm{pH} 6$ and incubated at $26 \mathrm{OC}$ for 10 days. The fungal biomass was separated by centrifugation at $15,000 \mathrm{rpm}$ for $10 \mathrm{~min}$. The obtained supernatant was subjected to liquid-liquid extraction with an equal amount of ethyl acetate in separating funnel. The organic layer was separated, then condensed to form crude and used as extracellular extract (Ex). The separated fungal mycelium was washed repeatedly to remove media components and dried at 60-80 OC in hot air oven. Then, the dried mycelium was crushed in pistil and mortar in order to extract intracellular secondary metabolites by adding ethyl acetate and centrifuged. The supernatant obtained was collected and evaporated to dryness. The obtained crude was used as intracellular extract (In). Approximately, the amount of crude extract from extracellular and intracellular solvent extraction yielded 0.7-0.8 $\mathrm{g} / \mathrm{L}$ and $0.25-0.30$ $\mathrm{g} / \mathrm{L}$, respectively. The both fungal extract and antifungal drugs of amphotericin $\mathrm{B}$, ketoconazole, fluconazole and nystatin were dissolved in $\mathrm{mg} / \mathrm{ml}$ of dimethyle sulfoxide (DMSO) as a test samples [20].

A Comparative Antifungal Activity of Fungal Crude Extracts with Standard antifungal Drugs
The antifungal activity was carried out against eight Candida strains of C. albicans 1966, C. albicans 2795, C. albicans 3019, C. glabrata 3814, C. glabrata 3981, C. tropicalis 1406, C. tropicalis 230 and $\mathrm{C}$. haemulonii 8303 by using agar well diffusion assay [21,22]. Sterilized yeast extract peptone dextrose agar medium (YPDA; Yeast extract-10g/l, peptone-20 g/l, dextrose-20 $\mathrm{g} / \mathrm{l}$, agar- $15 \mathrm{~g} / \mathrm{l}$ of distilled water, $\mathrm{pH}$ 6.8) was used to perform the antifungal activity. $24 \mathrm{~h}$ old freshly prepared Candida cultures were seeded on petriplate containing YPDA medium. Seven wells of each 5 $\mathrm{mm}$ in diameters were made with sterile cork borer. Two wells were loaded with each $50 \mu l$ of extracellular (Ex) and intracellular (In) test samples of $\mathrm{T}$. islandicus and other wells were loaded with standard drugs of amphotericin B, ketoconazole, fluconazole and nystatin, whereas DMSO was used as negative control and incubated at 28 OC for $24 \mathrm{~h}$. The efficacy of test samples and standard drugs was measured by zone of inhibition (ZOI) using Antibiotic Zone Scale-C PW297 (Himedia Laboratory Pvt. Ltd).

\section{Statistical analysis}

The biomass yield was recorded by drying the fungal mycelium at 60-80 OC and obtained weight was expressed as $\mathrm{g} / 100 \mathrm{ml}$ of culture broth. The antifungal activity of standard antifungal drugs and extract of $T$. islanicus were assessed by means \pm SD (Standard deviation); using Microsoft Excel.

\section{Results and Discussion}

\section{Optimization of Growth Parameters}

The microbial optimization process is required to check the effect of various nutrients and physical environmental factors on production of novel metabolites having various biological activities are mainly based on particular strain to be used. During optimization, the both solid state fermentation (SSF) and submerged fermentation (SMF) methods were employed for extraction of secondary metabolites. The solid-state fermentation (SSF) was not much supports for the secondary metabolite production, whereas highest metabolite yield was observed in submerged fermentation (SMF). Noura et al., successfully employed the production of $\beta$ glucosidase through solid state fermentation by species Talaromyces pinophilus, thus the fermentation process is largely depends on the type of strain to be used [23]. The basic production of fungal bioactive products has largely been produced by liquid based submerged fermentation have been reported (SMF) [24]. However, conventional technology for the production of valuable fungal products is mainly obtained in liquid 

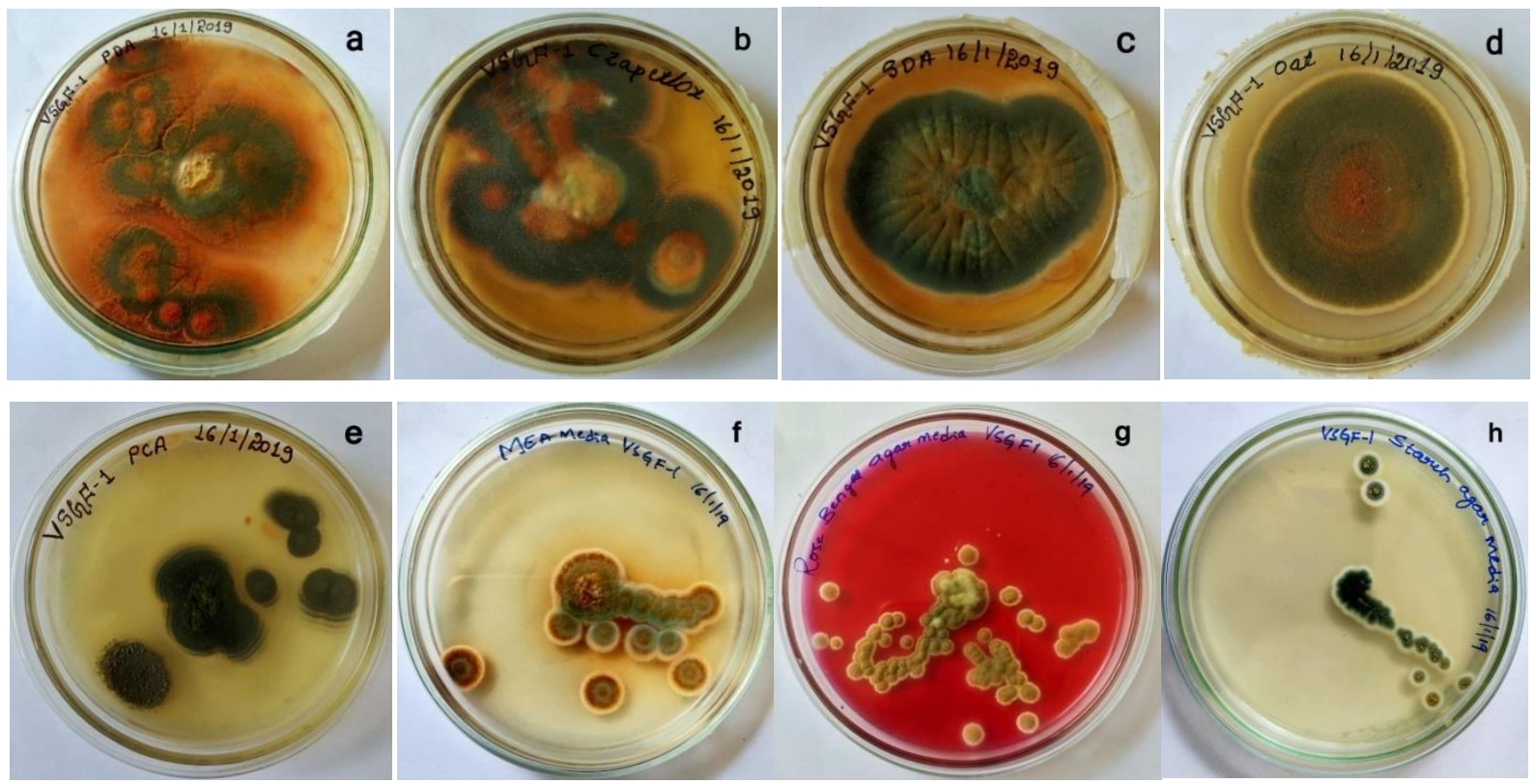

Figure 2. The mycelium growth and sporulation of T. islandicus VSGF1 on different nutrient media after 7 days of incubation period was observed on a) Potato dextrose agar b) Czapek dox agar c) Sabouraud dextrose agar d) Oat meal e) Potato carrot agar f) Malt extract agar g) Martin rose Bengal agar and h) Starch agar.

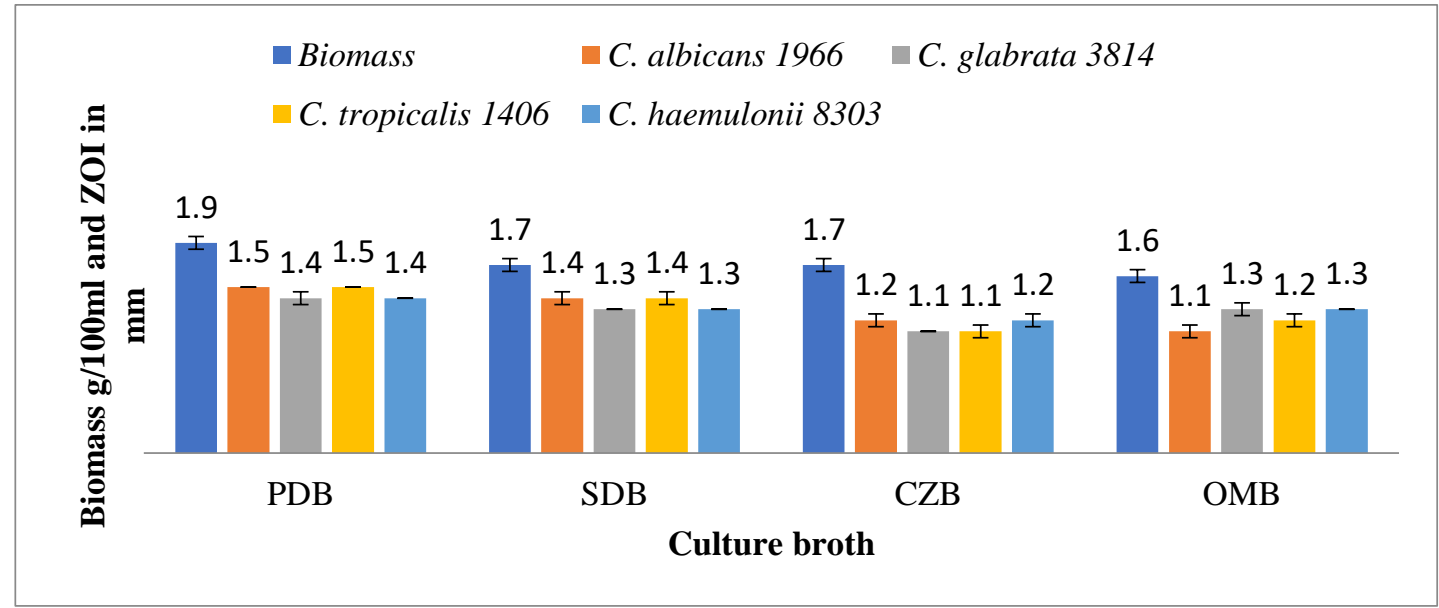

Figure 3. Effect of different broth cultures on production of fungal biomass and antifungal activity against Candida spp. [Data expressed as mean $\pm \operatorname{SD}(n=3)]$.

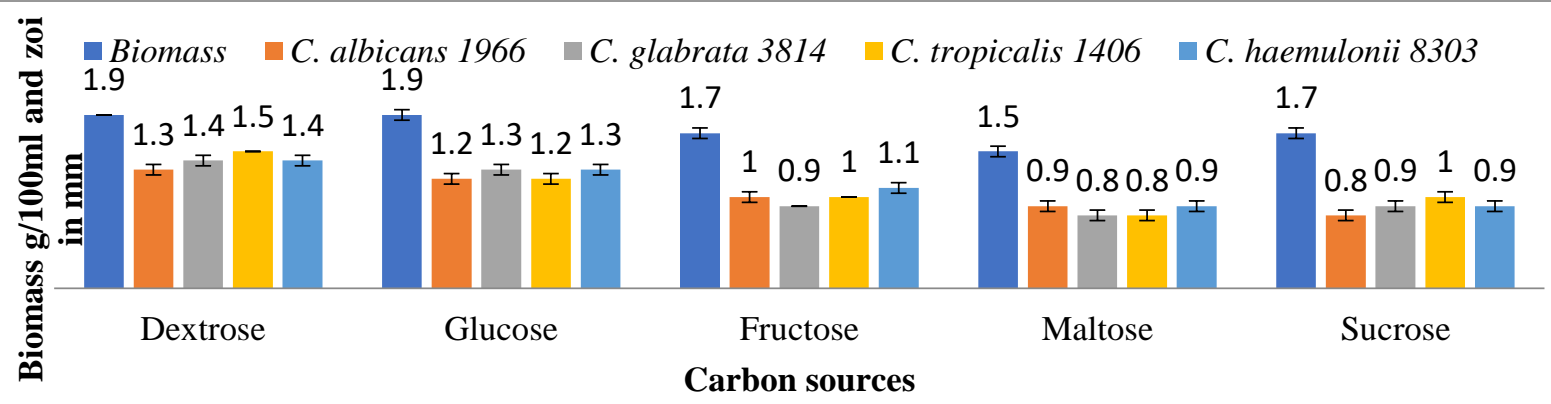

Figure 4. Effect of simple and complex carbon sources were studied on production of fungal biomass and antifungal activity against Candida spp. [Data expressed as mean $\pm S D(n=3)$ ]. 
submerged fermentation [25]. Hence, the SMF method was employed for the extraction of crude extract throughout the studies.

\section{Optimization of Culture Media}

Initially, the culture medium was optimized for the maximum production of biomass and highest yield of antifungal secondary metabolites against human pathogenic Candida spp. Among the eight media used, potato dextrose agar medium was found suitable for the fast and better growth of $T$. islandicus with characteristic soluble pigment as compared to other media. Whereas, czapekdox medium, sabouraud dextrose medium and oatmeal medium also enhanced the better growth of mycelium then next to PDA medium (Figure 2a, b, c \& d). Whereas, the poor and slow growth was observed in potato carrot agar, malt extract agar, Martin rose Bengal agar and starch agar (Fig. 2e, f, $g \& h$ ). Mikio et al., reported that, T. bacillosporus was grown on medium amended with malt extract supports the pigment extraction along with antibacterial activity [26]. In present studies, the malt extract containing medium enhances the pigment production with reduced amount of biomass as well as antifungal activity. The effect of PDA medium on fungal growth revealed a strong exponential growth up to 7 days and reached to stationary phase on 9th day of incubation period. The highest secondary metabolite secretion was observed in medium supplemented with dextrose and maximum biomass was obtained in media containing sucrose along with supplementary substances like dipotassium phosphate, ferrous sulfate, magnesium, potassium chloride and oatmeal. Similarly, among the broth used, potato dextrose broth (PDB) produced highest $1.9 \mathrm{~g}$ of biomass yield and followed by sabouraud dextrose broth (SDB) $1.7 \mathrm{~g}$, czapek dox broth (CZB) $1.7 \mathrm{~g}$ and Oat meal (OMB) $1.6 \mathrm{~g} / 100 \mathrm{ml}$ of culture broth. As compared to antifungal activity, the highest $15 \mathrm{~mm}$ ZOI was recorded by PDB and followed by SDB $14 \mathrm{~mm}$ against $C$. albicans 1966 and $C$. tropicalis 1406 . The $\mathrm{OMB}$ and CZB extract showed $13 \mathrm{~mm}$ and $12 \mathrm{~mm}$ against C. glabrata 3814 and C. haemulonii 8303, respectively (Figure $\mathbf{3}$ ). The variation observed in fungus growth and activity among the broth used is due to utilization of nutrients or may be related to media composition in which the fungus was grown. The similar studies were reported with maximum mycelium weight and inhibitory bioactive metabolites in potato dextrose broth against Klebsilla oxytoca, Staphylococcus aureus and Bacillus subtilis, respectively $[15,27]$. The maximum biomass and highest activity of $T$. islandicus VSGF1 was achieved significantly in potato dextrose medium, hence the medium was further used to optimize other cultural parameters for better growth and production of antifungal secondary metabolites.

\section{Effect of Carbon Sources}

Among the carbon sources used, the highest $1.9 \mathrm{~g} / 100 \mathrm{ml}$ of fungal biomass and significant antifungal activity was achieved in basal medium amended with $2 \%$ dextrose followed by glucose. During the optimization studies, both simple and complex carbon sources were examined and found highest of $15 \pm 0 \mathrm{~mm}$ ZOI against C. tropicalis 1406 in media supplemented with dextrose (Figure 4). The low activity was recorded in medium supplemented with maltose. The glucose at $2 \%$ concentration also yielded $1.9 \mathrm{~g} / 100 \mathrm{ml}$ of biomass and showed reduced $\mathrm{ZOI}$ of $13 \pm 0.057 \mathrm{~mm}$ against C. glabrata 3814 and C. haemulonii 8303 as compared to dextrose. In the present investigation, the $2 \%$ carbon concentration was optimum for the growth of fungal biomass and antifungal activity was also found maximum at similar concentration. Carbon sources are major nutritive components and building blocks of overall growth and development of microbes and each carbon sources play a different role thus enhances the production of novel secondary metabolites. Asnaashari et al., who has reported the highest production of antibiotic penicillin was achieved in medium supplemented with lactose as a carbon source [16]. Verma et al., reported that, the endophytic species of Aspergillus isolated from Calotropis procera showed the highest antimicrobial metabolites in culture medium supplemented with starch [28].

\section{Effect of nitrogen Sources}

In the present optimization process, the nitrogen sources have found a negative impact on production of fungal biomass along with antifungal activity. Among the nitrogen sources used, quite better activity was recorded from yeast extract as compared to peptone. The medium supplemented with yeast extract showed the reduced amount of biomass $1.2 \mathrm{~g} / 100 \mathrm{ml}$ and $9 \pm 0.057 \mathrm{~mm}$ ZOI against C. haemulonii 8303 (Figure 5). As the concentration of nitrogen sources increased, the fungal biomass decreased. In the present study, only carbon source alone supported for the production of biomass as well as antifungal metabolites. It has been also reported that some nitrogen sources have inhibitory action on production of some secondary metabolites and also antimicrobial agents due to sugar catabolic repression and acid accumulation resulting in oxygen depletion and $\mathrm{pH}$ imbalance. The optimization studies reported on Aspergillus sp. CRP5 showed $3 \mathrm{~g} / \mathrm{L}$ of yeast extract influences the secondary metabolite production [28]. The utilization of nitrogen source is generally depends on the type of microorganism, most of the microbes 


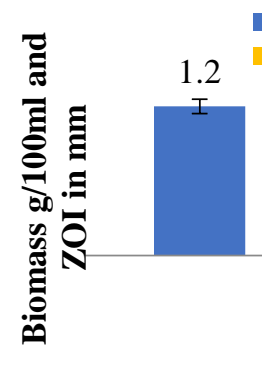

- Biomass

C. tropicalis 1406

C. albicans 1966

C. glabrata 3814

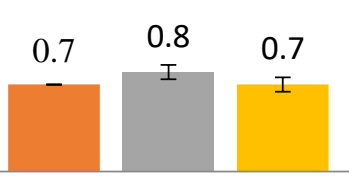

Yeast extract
C. haemulonii 8303

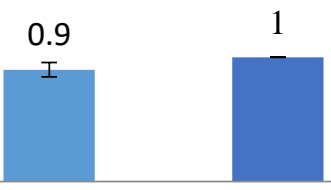

0.6

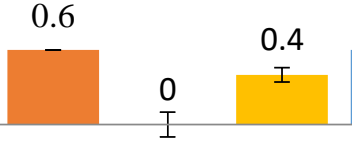

0.6

Peptone

\section{Nitrogen sources}

Figure 5. Effect of various nitrogen sources were investigated on production of fungal biomass and antifungal activity against pathogenic Candida spp. [Data expressed as mean \pm SD $(n=3)$ ].

$\square$ Biomass $\square$ C. albicans $1966 \backsim$ C. glabrata $3814 \square$ C. tropicalis $1406 \square$ C. haemulonii 8303

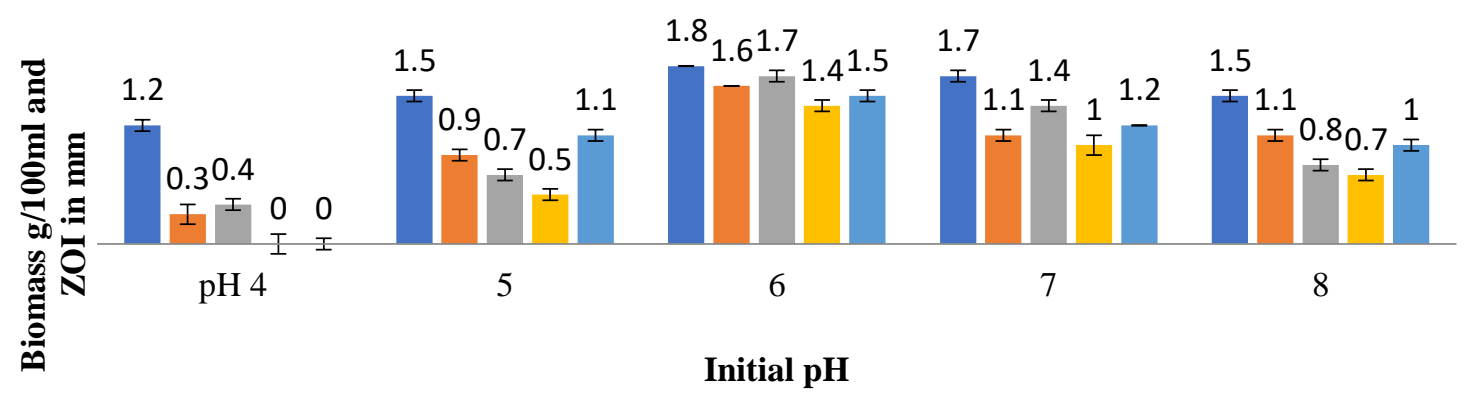

Figure 6. Effect of different $\mathrm{pH}$ was determined for maximum production of fungal biomass and highest antifungal activity against Candida spp. [Data expressed as mean $\pm S D(n=3)$ ].

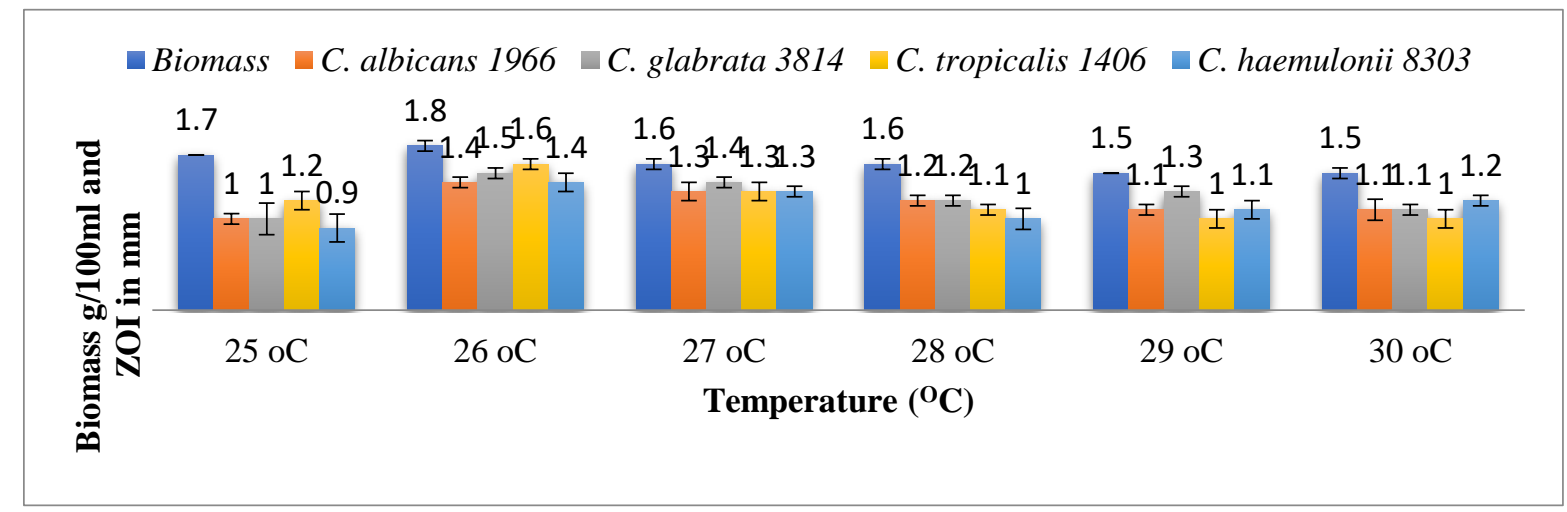

Figure 7. Effect of temperature ranges from 25 to $30 \mathrm{OC}$ was studied on production of fungal biomass and antifungal activity against Candida spp. [Data expressed as mean \pm SD $(n=3)$ ].

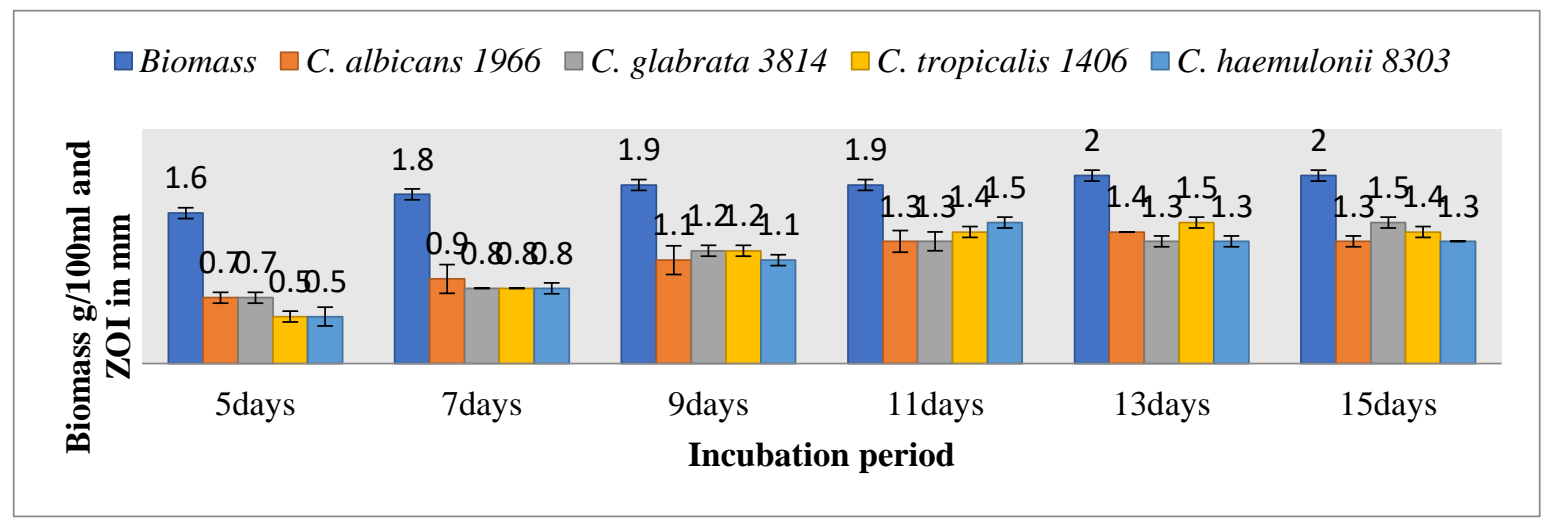

Figure 8. Effect of incubation period from 5 to 15 days at difference of one was recorded on production of fungal biomass and antifungal activity against Candida spp. [Data expressed as mean \pm SD $(n=3)$ ]. 
metabolized the nitrogen sources to produce various cell components [29]. The remarkable characteristic feature of fungi is their extraordinary flexibility with respect to carbon and nitrogen sources. The requirement of carbon and nitrogen $(\mathrm{C}: \mathrm{N})$ ratio varied among the fungal species, thus the growth characteristics might be strain dependent [30].

\section{Effect of $\mathrm{pH}$}

The $\mathrm{pH}$ of medium plays a vital role in the overall growth and development of micro-organisms. The $\mathrm{T}$. islandiucs was grown at varying $\mathrm{pH}$ from 4 to 8 at difference of one and found maximum $1.8 \mathrm{~g} / 100 \mathrm{ml}$ of biomass production along with, the highest of $17 \pm 0.057 \mathrm{~mm}$ and $16 \pm 0 \mathrm{~mm}$ ZOI formed against C. glabrata 3814 and C. albicans 1966, respectively at pH 6 (Figure 6). In case of $\mathrm{pH} 4$ the poor growth was observed, whereas at $\mathrm{pH} 5$ and 8 the biomass production was very less and produced a small rounded white colony about 3-5 $\mathrm{mm}$ in diameter. The $\mathrm{pH} 6$ of the basal medium was found to be maximum growth with higher antifungal activity and near to neutral $\mathrm{pH} 7$ also influenced the biomass growth and support the antifungal activity after $\mathrm{pH} 6$. A slight variation in $\mathrm{pH}$ of the medium significantly reduces the metabolite production and affects the cellular process such as biosynthesis and regulation of secondary metabolites [31]. Hence, the $\mathrm{pH}$ is most important in determining cultural parameter due to permeability characteristic of the cell wall and cell membranes are relatively depends on cell environment $\mathrm{pH}$ level. Similar studies were reported by Gogoi et al., on Fussarium solani produced maximum secondary metabolites at $\mathrm{pH} 6$ and other studies also supports the present investigation $[32,33,34]$. Most of the fungal strain has its specific $\mathrm{pH}$ in which it grows and reproduces. Generally, fungi prefer an acidic $\mathrm{pH}$ for good growth and activity. Usually too alkaline and too acidic solutions showed a negative impact on fungal growth and $\mathrm{pH}$ from 4 to 9 is favorable for the growth of fungi $[35,36]$ and change in $\mathrm{pH}$ of the medium induces the production of new metabolite may lead to adverse effect on antibiotic production.

\section{Effect of temperature}

$T$. islandicus was grown at temperature ranges between 25 to $30 \mathrm{OC}$ at a difference of one to the basal medium. The maximum fungal biomass $1.8 \mathrm{~g} / 100 \mathrm{ml}$ and significant antifungal activity of $16 \pm 0.057 \mathrm{~mm}$ was found against C. tropicalis 1406 at $26 \mathrm{OC}$ (Figure 7). Whereas, at temperature $25 \mathrm{OC}$ also supports the maximum production of biomass with less antifungal activity and vice-versa 27 OC showed highest activity with less biomass production. Thus, the ttemperature has profound effect not only in the production of metabolites as well as in the morphological and physiological aspects of the micro-organisms [37]. Similar studies were reported by Ritchie et al., and Jain et al., produced maximum growth and highest antimicrobial activity at temperature 25 OC during the optimization of Rhizoctonia solani and Aspergillus terreus [18,33]. In most optimization studies, the temperature 26 OC was optimum for the production of bioactive secondary metabolites and supports the good growth of fungal biomass [28]. Compaore et al., reported that the highest production of antimicrobial substances by Aspergillus fumigates was recorded at temperature 37 OC [19]. When temperature below 20 OC, less biomass production and low activity was reported and above $40 \mathrm{OC}$ fungal growths was stopped, thus the low temperature may reduce the metabolic activity and high temperature kills the fungal cells [32].

\section{Effect of incubation period}

The production of maximum $2 \mathrm{~g} / 100 \mathrm{ml}$ of biomass was recorded on 13th and 15th day of incubation period, whereas the highest antifungal activity of $15 \pm 0.057 \mathrm{~mm}$ ZOI was recorded from 9th, 13th and 15 th day of incubation period against $C$. heamulonii 8303, C. tropicalis 1406 and C. albicans 3019, respectively (Figure 8). As the incubation period increases the production of biomass increased, whereas the antifungal activity remains the same as the 9 to 15th day of incubation. Hence, the suitable incubation time for the production of highest biomass and antimicrobial secondary metabolites was observed at 9th day. The incubation period directly affects the total growth and overall biological process of the fungi and the fungus which was incubated for 9-10 days produced maximum secondary metabolites in provided culture medium have been reported [28]. The fungal biomass and production of secondary metabolites remain constant after 10 days of incubation period.

\section{Effect of agitation}

The $T$. islandicus was subjected to different agitation speed and showed maximum $1.8 \mathrm{~g} / 100 \mathrm{ml}$ of biomass production with $14 \pm 0.057 \mathrm{~mm}$ ZOI against C. haemulonii 8303 at $160 \mathrm{rpm}$ (Fig. 9). However, the highest of $16 \pm 0.057 \mathrm{~mm} \mathrm{ZOI} \mathrm{against} C$. tropicalis 1406 along with $1.9 \mathrm{~g} / 100 \mathrm{ml}$ of fungal biomass production was observed in culture broth kept under stationary condition. Agitation helps in equal distribution of nutrients and aeration to increase the rate of metabolites in the fermentation medium and it regulates the oxygenation rate in an Erlenmeyer flask. The high agitation may damage the fragmented hyphae and affects the cellular metabolism, thus consequently reduce the antibiotic production [19]. In the present study, the 
$\square$ Biomass $\square$ C. albicans $1966 \square$ C. glabrata $3814 \square$ C. tropicalis $1406 \square$ C. haemulonii 8303

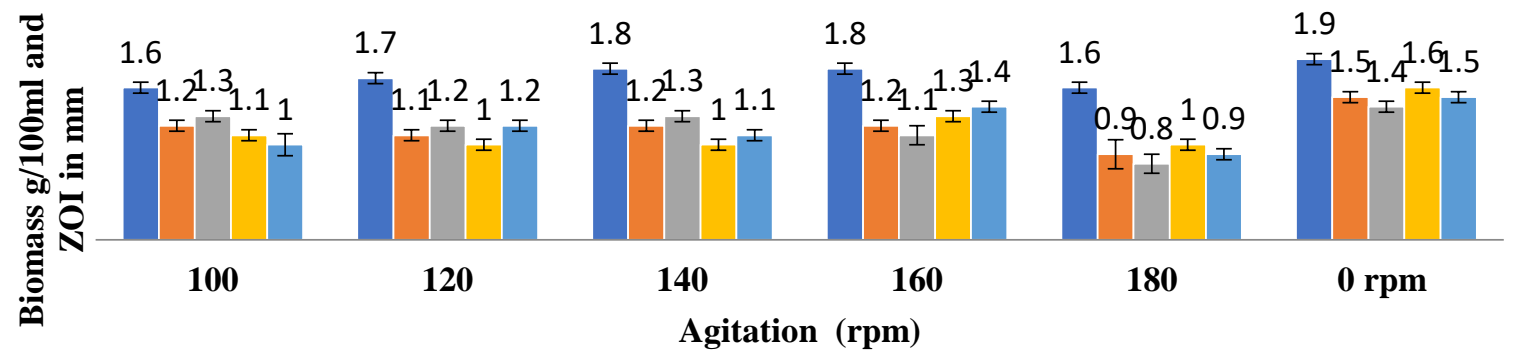

Figure 9. Effect of different agitation speed along with control static condition was assessed on production of fungal biomass and antifungal activity against Candida spp. [Data expressed as mean $\pm \operatorname{SD}(n=3)$ ].
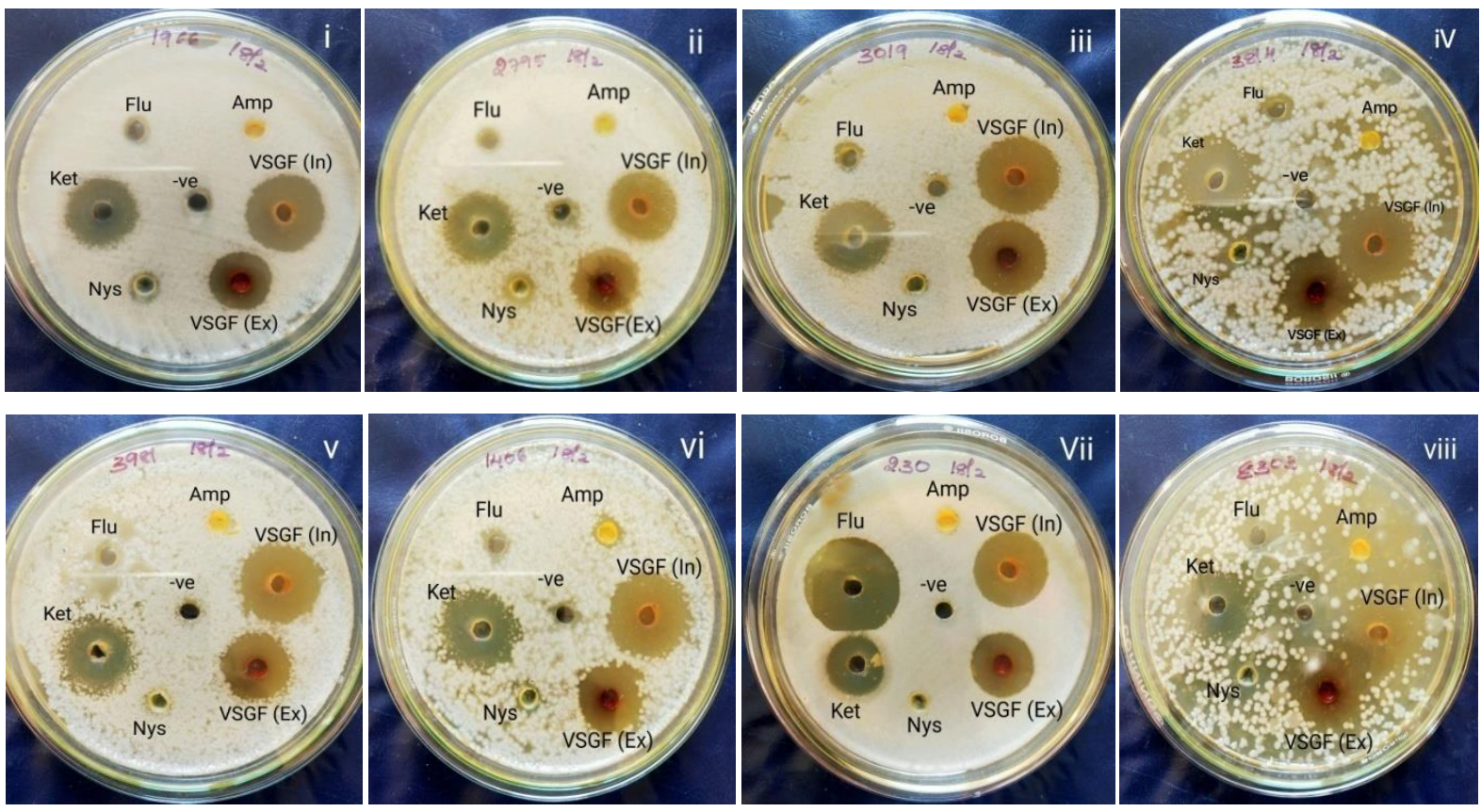

Figure 10. Antifungal activity of $T$. islandicus VSGF1 [VSGF (In) - intracellular extract of T. islandicus VSGF1; VSGF (Ex) - extracellular extract of T. islandicus VSGF1; -ve-negative control (DMSO)] with standard antifungal drugs [AmpAmphatericin B, Flu-Fluconazole, Ket-Ketoconazole, Nys-Nystatin] showing zone of inhibition against tested Candida spp. i) C. albicans MTCC1966, ii) C. albicans MTCC2795 iii) C. glabrata MTCC3019 iv) C. glabrata MTCC3814 v) C. glabrata MTCC3981 vi) C. tropicalis MTCC1406 vii) C. tropicalis MTCC230 viii) C. haemulonii MTCC8303

Table 1. A Comparative antifungal activity of Talaromyces islandicus VSGF1 with standard antifungal drugs

\begin{tabular}{|c|c|c|c|c|c|c|}
\hline Candida spp. MTCC & Amp-B & Flu & Ket & Nys & $\begin{array}{c}\text { T. islandicus } \\
\text { VSGF (In) }\end{array}$ & $\begin{array}{c}\text { T. islandicus } \\
\text { VSGF (Ex) }\end{array}$ \\
\hline C. albicans 1966 & --- & --- & $14.33 \pm 0.57$ & --- & $16.66 \pm 0.57$ & $13.33 \pm 0.57$ \\
\hline C. albicans 2795 & --- & --- & 14 & --- & $15.33 \pm 0.57$ & $13.33 \pm 0.57$ \\
\hline C. albicans 3019 & --- & --- & $14.33 \pm 0.57$ & --- & $16.33 \pm 0.57$ & $15.66 \pm 0.57$ \\
\hline C. glabrata 3814 & --- & --- & $13.33 \pm 0.57$ & --- & $17.66 \pm 0.57$ & $13.66 \pm 0.57$ \\
\hline C. glabrata 3981 & --- & --- & $13.33 \pm 0.57$ & --- & $16.33 \pm 0.57$ & $13.33 \pm 0.57$ \\
\hline C. tropicalis 1406 & --- & --- & $13.33 \pm 0.57$ & --- & 15 & $13.33 \pm 0.57$ \\
\hline C. tropicalis 230 & --- & $19.33 \pm 0.57$ & $12.33 \pm 0.57$ & --- & 15 & $14.33 \pm 0.57$ \\
\hline C. haemulonii 8303 & --- & --- & $13.33 \pm 0.57$ & --- & $15.33 \pm 0.57$ & $13.33 \pm 0.57$ \\
\hline
\end{tabular}

*Note: Amp-B: Amphatericin B, Flu- Fluconazole, Ket-Ketoconazole, Nys-Nystatin, Ex-Extracellular, In-Intracellular $[n=3 ;$ mean $\pm S D]$. 
maximum amount of biomass and antimicrobial substances were produced at stationary condition.

The current studies on optimization of growth parameters influenced the maximum production of fungal biomass as well as the highest antifungal secondary metabolites. Initially, when the $T$. islandicus was grown in potato dextrose broth at $\mathrm{pH}$ 6.8 and temperature $28 \mathrm{OC}$ produced maximum $1.7 / 100 \mathrm{ml}$ of biomass and highest of $14 \mathrm{~mm} \mathrm{ZOI}$ against tested Candida spp. Whereas, after the optimization of PDB, the production of fungal biomass increased 1.9 to $2 \mathrm{~g} / 100 \mathrm{ml}$ and antifungal activity from $14 \mathrm{~mm}$ to $17 \mathrm{~mm}$, thus showed the promising results against drug resistant Candida spp., as compare to frontline antifungal drugs. Hence, in the present investigation the $\mathrm{pH}$ and temperature played a major role in enhancement of the highest secondary metabolite production in the culture broth.

\section{A Comparative Antifungal Activity of Fungal Crude Extracts with Standard Drugs}

The antifungal activity of extracellular and intracellular crude extract of $T$. islandicus VSGF1 showed promising results by exhibiting highest activity when compared to standard drugs of amphotericine B, fluconazole, ketoconazole and nystatin. The ethyl acetate crude intracellular extract showed highest of $17.66 \pm 0.57 \mathrm{~mm}$ ZOI against C. tropicalis 1406 and followed by $16.66 \pm 0.57 \mathrm{~mm}$ against C. albicans 1966, C. albicans 3019 and C. glabrata 3981. Whereas, the extracellular extract exhibited maximum of $15.66 \pm 0.57 \mathrm{~mm}$ ZOI higher than that of the standard ketoconazole 14.66 \pm 0.57 $\mathrm{mm}$ against, C. albicans 1966, C. albicans 3019, C. tropicalis 1406 and C. tropicalis 230 (Table 1 \& Figure 10). The intracellular extract of $T$. islandicus showed highest antifungal activity whereas extracellular extract exhibited equal activity when compare to ketoconazole.

Among the standard drugs, only ketoconazole was found susceptible to all tested Candida species with significant inhibitory activity. However, fluconazole exhibited susceptibility only against the single test organism C. tropicalis 230 with $19.33 \pm 0.57 \mathrm{~mm} \mathrm{ZOI}$. The results revealed the development of resistance to tested Candida species at drug concentrations of $\mathrm{mg} / \mathrm{ml}$ of amphotericine $\mathrm{B}$, fluconazole and nystatin. Interestingly, at the same concentration of extracellular and intracellular crude extract of $T$ islandicus VSGF1 showed significant inhibitory activity against all tested Candida spp. All the antifungal agents tested in the present study have a documented clinical effect on Candida spp. In present study, the Candida species exhibited resistant to amphotericin- $B$, fluconazole and nystatin is may be due to continue screening of clinical isolates to the antifungal drugs may sometimes lead to development of resistance among the Candida. Overuse of azole drugs has an important role in the development of fluconazole resistance in Candida spp. This resistance has an increasing trend among C. albicans and non-albicans Candida species over recent decades [11]. However, C. glabrata is highly resistant to fluconazole, with a global resistance rate of $15.7 \%$ as compared to other Candida species. Primary resistance to fluconazole is rare in $\mathrm{C}$. albicans (1.4\%) and C. tropicalis (4.1\%) [38]. Nolte et al., reported the isolation of AmB-resistant in C. albicans from patients with leukemia [39]. The lack of activity of $A m B$ is described in systemic murine fusarial infection as well as in clinical isolates $[40,41]$. Powderly et al., noted that there is a correlation between failure of $A m B$ therapy and in vitro resistance in yeasts isolated from cancer patients with invasive fungal infection [42]. Azoles (fluconazole and ketoconazole) and polyenes (amphotericin-B and nystatin) are the two most frontline antifungal drugs in hospitals and increasing resistance to both frontline antifungals severely hampers our ability to treat fungal disease. Combination therapy could be a promising method to increase the effectiveness of antimicrobial agents. The combination of two or more antifungal drugs or non-antifungal drugs has been appraised towards to overcome the therapeutic failures $[43,44]$. This necessitates the search for novel bioactive metabolite with potential antimicrobial agent. The fungus $T$. islandicus extracts alone had a stronger effect against tested Candida isolates than its combination with common antifungal drugs and may lead to discovery of a strong and effective antifungal agent. However, this finding needs future studies to be further clarified.

The genus Talaromyces is well known for the production of effective antibacterial and antifungal secondary metabolites have been previously reported and thus support the present investigation $[45,46,47]$. The Talaroconvolutins and Talaroderxines obtained from T. convolutes and T. derxii exhibited inhibitory activity against Candida albicans and Bacillus subtilis, respectively $[48,49]$. The T. flavus strains have been reported to be effective against Verticillium, Aspergillus fumigates and C. albicans [50,51,52]. Hence, the current research was carried out on $T$. islandicus VSGF1 to screen against pathogenic Candida spp. and it has been confirmed that the $T$. islandicus is a potential soil fungus for the production of antifungal secondary metabolites against the broad spectrum of Candida spp.

\section{Conclusion}

The present investigation was successful in producing the highest biomass with the maximum 
antifungal activity of $T$. islandicus through optimization of growth parameters. The fungal crude extracts have shown promising antifungal activity against drug resistance Candida spp. and confirmed the growth inhibition in C. albicans, C. glabrata, C. tropicalis and C. haemulonii, who have developed multidrug resistance against frontline drugs of amphatericin B, fluconazole and nystatin. The results will serve as a base to identify the lead biomolecules useful in the production of new antifungal drugs to deal the infections caused by drug resistant Candida species. The bioactive metabolites produced by $\mathrm{T}$. islandicus VSGF1 proved to exhibit potent antifungal effect against pathogenic Candida species; hence these secondary metabolites would be used for the treatment of infections caused by above mentioned Candida species, after identifying its chemical nature and scientific validation through clinical studies.

\section{Acknowledgments}

The authors are grateful to the "Microbial Type Culture Collection (MTCC) and Gene Bank" Chandigarh, India for providing the Candida spp. for antifungal activity.

\section{Conflicts of interests}

Nil

\section{References}

1. Frisvad JC. Taxonomy, chemodiversity and chemoconsistency of Aspergillus, Penicillium and Talaromyces species. Front. Microbiol. 2015; $5(1): 1-7$.

2. Marino $T$, Nishimoto $M$, Masuda A, Fujita $S$, Nishikiori T, Saito S. NK374200, a novel insecticidal agent from Talaromyces, found by physico-chemical screening. J. Antibiot. 1995; 48(12):1509-10.

3. Thrane U, Rasmussen KB, Petersen B, Rasmussen $\mathrm{S}$, Sicheritz-Ponten, Mortensen UH. Genome sequence of Talaromyces atroroseus, which produces red colorants for the food industry. Genome Announc. 2017; 5(9):1736-16.

4. Hong LL, Xiao ML, Hui L, Ling HM, Bin G W. Two new diphenylketones and a new xanthone from Talaromyces islandicus EN-501, an endophytic fungus derived from the marine red algae Laurencia okamurai. Mar. Drugs. 2016; 14:1-8.

5. Sanchez JF, Somoza AD, Keller NP, Wang CCC. Advances in Aspergillus secondary metabolite research in the post-genomic era. Nat. Prod. Rep. 2012; 29(3):351-71.
6. Brakhage AA. Regulation of fungal secondary metabolism. Nat. Rev. Microbiol. 2013; 11:21-32.

7. Scherlach K, Graupner K, Hertweck C. Molecular bacteria-fungi interactions: effects on environment, food and medicine. Annu. Rev. Microbiol. 2013; 67:375-97.

8. Takahashi JA, Teles APC, Bracarense AAP, Gomes DC. Classical and epigenetic approaches to metabolite diversification in filamentous fungi. Phytochem. Rev. 2013; 12:773-89.

9. Bertrand S, Bohni N, Schnee S, Schumpp O, Gindro K, Wolfender JL. Metabolite induction via microorganism co-culture: a potential way to enhance chemical diversity for drug discovery. Biotechnol. Adv. 2014; 32(6):1180-204.

10. Marmann A, Aly A H, Lin W, Wang B, Proksch P. Co-cultivation - a powerful emerging tool for enhancing the chemical diversity of microorganisms. Mar. Drugs. 2014; 12(2):104362.

11. Berkow EL, Lockhart SR. Fluconazole resistance in Candida species: a current perspective. Infect. Drug. Resist. 2017; 10:237-45.

12. Gao J, Wang $H$, Li Z, Wong AHH, Wang YZ, Guo $Y$, Lin X, Zeng G, Liu H, Wang Y, Wang J. Candida albicans gains azole resistance by altering sphingolipid composition. Nat. Commun. 2018; 9(1):4495.

13. Deorukhkar SC, Roushani S. Fluconazole resistance in Candida species: ten years experience at a rural tertiary care teaching hospital in India. J. Infect. Dis. and Pathog. 2017; $1: 102$

14. Sachafhauser T, Wibberg D, Ruckert C, Winkler A, Flor $L$, van Pee $K H$, Fewer DP, Sivonen $K$, Jahn $L$, Ludwig-Muller J, Caradec T, Jacques P, Hujibers MM, van Berkel WJ, Weber T, Wohlleben W, Kalinowski J. Draft genome sequence of Talaromyces islandicus ("Penicillium islandicus") WF-38-12, a neglected mold with significant biotechnological potential. J. Biotechnol. 2015; 211:101-2.

15. Mathan S, Subramanian V, Nagamony S. Optimization and antimicrobial metabolite production from endophytic fungi Aspergillus KC 582297. Euro. J. Exp. Biol. 2013; 3:138-44.

16. Asnaashari M, Ghanbary MAT, Tazick Z. Optimization of penicillin $G$ production by Penicillium chrysogenum. Ann. Biol. Res. 2012; 3(12):5434-40.

17. Cao C, Li R, Wan Z, Liu W, Wang X, Qiao J, Wang $D$, Bulmer G, Calderone R. The effects of temperature, $\mathrm{pH}$, and salinity on the growth and dimorphism of Penicillium marneffei. Med. Mycol. 2007; 45(5):401-7.

18. Ritchie F, Bain RA, McQuilken MP. Effects of nutrient status, temperature and $\mathrm{pH}$ on mycelia growth, sclerotial production and germination of 
Rhizoctonia solani from potato. J. Plant. Pathol. 2009; 91(3):589-96.

19. Compaore H, Sawadogo HL, Guira F, Ware L Y, Samandoulougou S, Savadogo A, Dianou D, Traore AS. Optimization of antimicrobial compounds production by Aspergillus fumigates isolated from maize in Ouagadougou, Burkina Faso. Current Res. Microbiol. Biotechnol. 2016; 4(4):903-11.

20. Al-Shaibani ABA, Al-Shakarchi FI, Ameen RS. Extraction and characterization of antibacterial compound from Aspergillus niger. Al-Nahrain J. Sci. 2013; 16(4):167-74.

21. Magaldi S, Mata-Essayag S, Capriles CH, Perez C, Colella MT, Olaizola C, Ontiveros Y. Well diffusion for antifungal susceptibility testing. Int. J. Infect. Dis. 2004; 8(1):39-45.

22. Valgas C, de Souza SM, Smania EFA, Smania A. Screening methods to determine antibacterial activity of natural products. Braz. J. Microbiol. 2007; 38(2):369-80.

23. Noura NEE, Haroun SA, Oweis EA, Sherief AA. Identification of newly isolated Talaromyces pinophilus and statistical optimization of $\beta$ Glucosidase production under solid state fermentation. Preparative Biochem. Biotechnol. 2015; 45(7): 712-29.

24. Gowthaman MK, Krishna C, Moo-Young M. Fungal solid state fermentation-an overview. Appl. Mycol. Biotechnol. 2001; 1:305-52.

25. Barrios-Gonzalez J, Tarrago-castellanos MR. Solid-State fermentation: special physiology of fungi. In: Merillon JM., Ramawat K. (eds) Fungal metabolites. References series in phytochemistry. Springer, Cham. 2017; 319-347.

26. Yamazaki M, Okuyama E. Isolation and structure of oxaphenalenone dimmers from Talaromyces bacillosporus. Chem. Pharm. Bull. 1980; 28(12): 3649-55.

27. Bhattacharyya PN, Jha DK. Optimization of cultural conditions affecting growth and improved bioactive metabolite production by a subsurface Aspergillus strain TSF 146. Int. J. Appl. Biol. Pharm. Technol. 2011; 2(4):133-45.

28. Verma SK, Lal M, Debnath M. Optimization of process parameters for production of antimicrobial metabolites by endophytic fungus Aspergillus sp. CPR5 isolated from Calotropis procera root. Asian J. Pharma. Sci. Clin. Res. 2017; 10(4):225-30.

29. Mustafa U, Kaur G. Effects of carbon and nitrogen source and ratio on the germination, growth and sporulation characteristics of Metarhizium anisopliae and Beauveria bassiana isolates. Afr. J. Agric. Res. 2009; 3(10):922-30.

30. Gao L, Sun MH, Liu XZ, Che YS. Effects of carbon concentration and carbon to nitrogen ratio on the growth and sporulation of several bio-control fungi. Mycol. Res. 2007; 111(1):87-92.

31. Hassan SAA, Bakhiet SEA. Optimization of antibacterial compounds production by Aspergillus fumigates isolated from Sudanese indigenous soil. Int. Biol. Biomed. J. 2017; 3(4):204-8.

32. Gogoi DK, Deka Boruah HP, Saikia R, Bora TC. Optimization of process parameters for improved production of bioactive metabolite by a novel endophytic fungus Fusarium sp. DF2 isolated from Taxus wallichiana. World J. Microbiol. Biotechnol. 2008; 24(1):79-87.

33. Jain P, Pundir RK. Effect of fermentation medium, $\mathrm{pH}$ and temperature variations on antibacterial soil fungal metabolite production. Int. J. Agric. Technol. 2011; 7(2):247-69.

34. Palanichamy P, Krishnamoorthy G, Kannan S, Marudhamuthu $M$. Bioactive potential of secondary metabolites derived from medicinal plant endophytes. Egypt. J. Basic and Appl. Sci. 2018; 5(4):303-12.

35. Meier CL, Rapp J, Bowers RM, Silman M, Fierer N. Fungal growth on a common wood substrate across a tropical elevation gradient: Temperature sensitivity, community composition, and potential for above-ground decomposition. Soil Biol. Biochem. 2010; 42(7):1083-90.

36. Sharma A, Sharma M, Chandra S. Influence of temperature and relative humidity on growth and sporulation of some common dermatophytes. Indian J. Fund. Appl. Life Sci. 2012; 2(4):1-6.

37. Vijayakumar R, Panneerselvam K, Muthukumar C, Thajuddin N, Paneerselvam A, Saravanamuthu R. Optimization of antimicrobial production by a marine actinomycetes Streptomyces sfghaniensis VPTS3-1 isolated from palk strait, east coast of India. Indian J. Microbiol. 2012; 52(2): 230-39.

38. Pfaller MA, Diekema DJ, Gibbs DL, Newell VA, Ellis D, Tullio V, Rodloff A, Fu W, Ling TA. Results from the ARTEMIS DISK Global Antifungal Surveillance Study, 1997 to 2007: a 10.5- year analysis of susceptibilities of Candida species to fluconazole and voriconazole as determined by CLSI standardized disk diffusion. J. Clin. Microbiol. 2010; 48(4):1366-77.

39. Nolte FS, Parkinson T, Falconer DJ, Dix S, Williams J, Gilmore C, Eller R, Wingard JR. Isolation and characterization of fluconazole- and amphotericin B-resistant Candida albicans from blood of two patients with leukemia. Antimicrob. Agents Chemother. 1997; 41(1):196-9.

40. Anaissie EJ, Hachem R, Legrand C, Legenne P, Nelson P, Bodey GP. Lack of activity of amphotericin $B$ in systemic murine fusarial infection. J. Infect. Dis. 1992; 165(6):1155-7.

41. Ellis D. Amphotericin B: spectrum and resistance. J. Antimicrob. Chemother. 2002; 49(1):7-10. 
42. Powderly WG, Kobayashi GS, Herzig GP, Medoff G. Amphotericin B-resistant yeast infection in severely immunocompromised patients. Am. J. Med. 1988; 84(5):826-32.

43. Ahmadi A, Mahmoudi S, Rezaie S, Hashemi SJ, Dannaoui E, Badali H, Ghaffari M, Aala F, Izadi A, Maleki A, Meis JF, Khodavaisy S. In vitro synergy of echinocandins with triazoles against fluconazole-resistant Candida parapsilosis complex isolates. J. Glob. Antimicrob. Resist. 2020; 21:313-334.

44. Ahangarkani F, Khodavaisy S, Mahmoudi S, Shokohi T, Rezai MS, Fakhim H, Dannaoui E, Faraji $\mathrm{S}$, Choedhary A, Meis JF, Badali H. Indifferent effect of nonsteroidal anti-inflammatory drugs (NSAIDs) combined with fluconazole against multidrug-resistant Candida auris. Curr. Med. Mycol. 2019; 5(3):26-30.

45. Giang V, Tran H, Nguyen A, Nguyen Y, Nguyen T. Identification, antimicrobial activities and cytotoxicity of Talaromyces purpurogenus T013. J. Chem. Pharm. Res. 2017; 9(12): 174-9.

46. Pretsch A, Nagl M, Schwendinger K, Kreiseder B, Wiederstein M, Pretsh D, Genov M, Hollaus R, Zinssmeister D, Debbab A, Hundsberger H, Eger A, Proksch P, Wiesner C. Antimicrobial and antiinflammatory activities of endophytic fungi Talaromyces wortmannii extracts against acneinducing bacteria. PLoS ONE. 2014; 9(6).

47. Miao F, Yang R, Chen D, Wang Y, Qin B, Yang X, Zhou L. Isolation, identification and antimicrobial activities of two secondary metabolites of Talaromyces verruculosus. Molecules. 2012; 17: 14091-8.

48. Suzuki S, Hosoe T, Nozawa K, Kawai K, Yaguchi T, Udagawa S. Antifungal substances against pathogenic fungi, Talaroconvolutins, from Talaromyces convolutes. J. Nat. Prod. 2000; 63(6):768-72.

49. Suzuki S, Nozawa K, Nakajima S, Udagawa S, Kawai K. Isolation and structure of antibacterial binaphtho-a-pyrones, Talaroderxines $A$ and $B$, from Talaromyces derxii. Chem. Pharm. Bull. 1992; 40(5):1116-9.

50. Ayer WA, Racok JS. The metabolites of Talaromyces flavus: Part 2. Biological activity and biosynthetic studies. Can. J. Chem. 1990; 68(11):2095-101.

51. Komai S, Hosoe T, Tabashi T, Nozawa K, Okada K, Takaki GMC, Chikamori M, Yaguchi T, Fukushima K, Miyaji M, Kawai K. A new funicone derivative isolated from Talaromyces flavus IFM52668. Mycotoxin. 2004; 54(1):15-9.

52. Arai M, Tomoda H, Okuda T, Wang H, Tabata N, Masuma R, Yamaguchi Y, Omura S. Funicone related compounds, potentiators of antifungal miconazole activity produced by Talaromyces flavus FKI-0076. J. Antibiot. 2002; 55(2):172-80. 